\title{
Asteroseismic constraints on the OPAL opacity interpolation
}

\author{
W. M. Yang and M. Li \\ Department of Physics and Chemistry, Henan Polytechnic University, Jiaozuo 454000, China \\ email: yang.wuming@yahoo.com.cn
}

\begin{abstract}
There is a difference of a few Kelvins in the effective temperature between a model used only two-point interpolation of opacity and a model used piecewise linear interpolation of opacity. However the frequency difference between the models is of the order of several microHertz at a certain stage, which is almost 10 times worse than the observational precision of p-modes of solar-like stars. Therefore, the two-point interpolation of opacity is unsuitable in modelling of solar-like stars with element diffusion.
\end{abstract}

Keywords. stars: evolution, diffusion, stars: oscillations

Timescales of evolution and element diffusion are similar in solar-like stars with mass 1.0-1.5 $M_{\odot}$ (Turcotte et al. 1998). Thus element diffusion should be calculated in modelling of solar-like stars. Furthermore, helioseismology has confirmed the importance of including element diffusion and settling in solar modelling: models including effects of element diffusion and settling are better agreement with the seismically inferred sound-speed and density profiles than are models that ignored the effects (Christensen-Dalsgaard et al. 1993; Basu et al. 2000).

Heavy-element abundance, $Z$, is constant in main-sequence (MS) stage of solar-like stars without considering metal settling, thus evolution of these stars need only one set of opacity tables at a fixed $Z_{1}$. However the $Z$ is a variable in the models with metal settling and then the second set of opacity tables must be obtained at a fixed $Z_{2}$. Opacity at the desired $Z, X, T$, and $\rho$ can be obtained by two-point interpolation, i.e.,

$$
\kappa(Z, X, T, \rho)=\kappa_{2}\left(Z_{2}, X, T, \rho\right)+\frac{\kappa_{1}\left(Z_{1}, X, T, \rho\right)-\kappa_{2}\left(Z_{2}, X, T, \rho\right)}{Z_{1}-Z_{2}} \cdot\left(Z-Z_{2}\right),
$$

The value of $Z_{1}$ and $Z_{2}$ is chosen at a suitable value, respectively.

In order to study the impact of opacity interpolation, using the Yale Rotating Evolution Code (YREC7) in its nonrotating configuration, we construct four models listed in Table 1. All parameters of the models are same except the parameters of opacity interpolation. All models evolve from pre-main sequence (PMS) to somewhere near the end of the MS. The OPAL eos (Rogers \& Nayfonov 2002), OPAL opacity (Iglesias \& Rogers 1996), and the Alexander \& Ferguson(1994) opacity for low temperature are used. Element diffusion is implemented following the prescription of Thoul et al. (1994).

The changes in the effective temperature between our models at the same age are several Kelvin, which is within the error of observation of stellar effective temperature. The frequency differences between MT1 and MT2 are zero. In Fig. 1A, we represent the frequency differences between MT2 and MT3 at the same age. The difference increases, however, from about $1 \mu \mathrm{Hz}$ at the age of 1 Gyr to around $4 \mu \mathrm{Hz}$ at the age of 6 Gyr. But the difference at the age of 7 Gyr is less than that at the age of 6 Gyr. The frequency differences between MM1 and MT3 are shown in Fig. 1B. The frequency differences arrive at a maximum at the age of about 3 Gyr. Then with increase in age, 
Table 1. Model parameters.

\begin{tabular}{ccccc}
\hline Model & $\begin{array}{c}\text { Mass } \\
M \odot\end{array}$ & $Z_{0}$ & $\begin{array}{c}\text { Two-point interpolation } \\
Z_{1}--Z_{2}\end{array}$ & $\begin{array}{c}\text { Piecewise linear interpolation } \\
\delta z=Z_{i+1}-Z_{i}\end{array}$ \\
\hline MT1 & 1.10 & 0.022 & $0.022-0.021$ & $\ldots \ldots \ldots$ \\
MT2 & 1.10 & 0.022 & $0.022-0.020$ & $\ldots \ldots \ldots$ \\
MT3 & 1.10 & 0.022 & $0.022-0.019$ & $\ldots \ldots \ldots$ \\
MM1 & 1.10 & 0.022 & $\ldots \ldots \ldots \ldots \ldots \ldots \ldots$ & 0.001 \\
\hline
\end{tabular}

Notes: The $Z_{0}$ is the initial metal abundance. Piecewise linear interpolation: if $Z_{i} \leqslant Z<Z_{i+1}$, YREC will interpolate between $\kappa\left(Z_{i}\right)$ and $\kappa\left(Z_{i+1}\right)$ to obtain the opacity at the required $Z$.
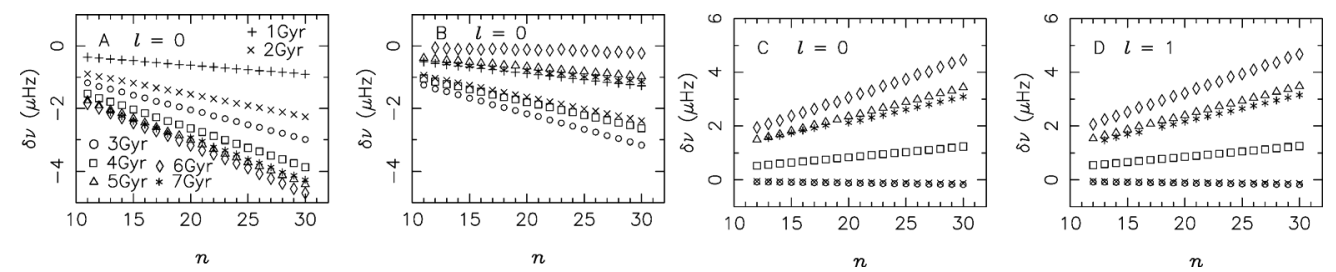

Figure 1. Frequency differences at different ages labeled by the different symbols. A: $\nu_{M T 2}-\nu_{M T 3}$; B: $\nu_{M M 1}-\nu_{M T 3}$; C and D: $\nu_{M M 1}-\nu_{M T 2}$.

the differences decrease. At the age of around $6 \mathrm{Gyr}$, the differences are almost zero. Thus the discrepancy between MM1 and MT3 mainly occurs between the ages of 2 and 4 Gyr. In Fig. 1C and D, we represent the frequency differences between MM1 and MT2. The differences are almost zero when the age of the models is less than 3 Gyr. Then with increase in age, the frequency differences increase. At the age of around 6 Gyr, the differences arrive at a maximum. The differences at the age of 4-7 Gyr are of the order of several microHertz, which is almost 10 times larger than the uncertainty of observation of stellar p-modes that is expected to reach 0.1-0.4 $\mu \mathrm{Hz}$ (Thèado et al. 2005; Bedding et al. 2004).

Heavy-element abundance is a constant in the models of PMS, and the $Z_{s}$ and $Z_{c}$ are close to the $Z_{0}$ in the early evolutionary stage of MS. Thus $Z_{1}$ is specified to be $Z_{0}$ in models MT1, MT2, and MT3, and one of $Z_{i}$ should be equal to $Z_{0}$ in MM1. The differences of the opacity interpolation in MT2, MT3, and MM1 should result in the difference between MT2, MT3, and MM1 and then lead the frequency difference between MT2, MT3, and MM1. The frequency difference between the models MT2, MT3, and MM1 is of the order of several microHertz at a certain phase, which is almost 10 times worse than the observational precision of p-modes of solar-like stars. Consequently, in modelling of solar-like star with metal settling, the two-point interpolation of opacity is unsuitable, and at least piecewise linear interpolation is required.

\section{References}

Alexander, D. R. \& Ferguson, J. W. 1994, ApJ, 437, 846

Basu, S., Pinsonneault, M. H., \& Bahcall, J. N. 2000, ApJ , 529, 1084

Bedding, T. R., Kjeldsen, H., Butler, P. R., et al. 2004, ApJ , 614, 380

Christensen-Dalsgaard, J., Proffitt, C. R. \& Thompson, M. J. 1993, ApJ , 403, L75

Iglesias, C. \& Rogers, F. J., 1996, ApJ, 464, 943

Rogers, F. J. \& Nayfonov, A, 2002, ApJ, 576,1064

Thado, S., Vauclair, S., Castro, M., Charpinet, S., \& Dolez, N. 2005, A\&A, 437, 553

Thoul, A. A., Bahcall, J. N., \& Loeb, A. 1994, ApJ, 421, 828

Turcotte, S., Richer, J., \& Michard, G. 1998, ApJ, 504, 559 